\title{
Moving Ship Detection Algorithm Based on Gaussian Mixture Model
}

\author{
Zuohuan Chen ${ }^{1}$, Jiaxuan Yang ${ }^{2, *}$ and Zhen Kang ${ }^{3}$ \\ ${ }^{1}$ Navigation College, Dalian Maritime University, Dalian 116026, China \\ ${ }^{2}$ The Key Laboratory of Navigation Safety Guarantee Liaoning Province, Dalian 116026, China \\ ${ }^{3}$ Navigation College, Dalian Maritime University, Dalian 116026, China \\ ${ }^{*}$ Corresponding author
}

\begin{abstract}
In order to reduce the influence of moving objects clutter in the background on the ship objects detection from ship video surveillance and improve the reliability of ship targets detection, this paper presents a method of ship objects detection using Gaussian mixture model. A Gaussian mixture model is established to estimate the background. The new pixel, in the video, which does not match the Gaussian distributions is regarded as foreground, otherwise background. The moving ship targets are detected by the continuity of the current and former frames, in which the foreground is obtained by subtracting the background from ship video. The target precision rate of the algorithm is $100 \%$ and the false alarm probability is $3.02 \%$ in the simulation experiment. Comparing with other algorithms, the results show that this algorithm can not only improve target precision rate, but also reduce false alarm probability, and greatly overcome the influence of large amount of clutter on the detection of moving ship objects in video background, effectively restraining the influence of the noise from the dynamic scenario transformation.
\end{abstract}

Keywords-traffic engineering; target detection; gaussian mixture model; moving ship; background subtraction; target segmentation

\section{INTRODUCTION}

With the development of shipping economy, the number of ships are growing rapidly, and make the water transportation industry increasingly busy, which increase the risk of ship accidents and threatens and affects the safety of the shipping personnel. Monitoring video installed on board, bridge, gate, etc. Video can dynamically analyzes real-time scenarios. By calculating the video information to detect the movement of the ship objects and its movement trend, and then command avoidance. It is a hot research topic to detect and track the ship targets, commanding and controlling the operation of the ship, which are based on video surveillance. Among them, the realtime detection and continuous tracking of the moving ship's targets are the primary links, and it is the basis of many followup studies, such as video motion analysis, behavior understanding and so on.

Comparing with other monitoring systems, the maritime video surveillance has the following characteristics: background clutter, ship video surveillance background contains a lot of shaking water ripples and light spots affecting the ship objects detection; Camera shaking: result in a non-linear change in the state of the target; Illumination variations: different seasons, time periods, illumination intensity and direction affect the detection of ship targets directly.

At present, there are many methods for the detection of video moving target, and there are adjacent frame difference method (AFDM) [1] and optical flow method [2] in the common moving target detection method. Though AFDM has a strong adaptability to the change of illumination, scale and other testing environment. It is easy to detect holes in the target of moving ships when dealing with the texture of the target pixel of the moving ship and the change of the color gradation. Optical flow method can independently detect the ship target, doesn't need to know in advance video ship target motion scenario, but this method needs a large number of calculations and has poor antinoise performance, which is difficult to meet the requirements of real-time detection.

Background modeling plays a greatly role in ship target detection by using the background subtraction. Friedman et al., (1997) established a single Gaussian distribution model, believing that each pixel value in the video image approximately obeyed Gaussian distribution and by using the obtained pixel value to estimate Gaussian distribution parameters [3]. The new pixels, in the video, which did not match the Gaussian distributions was regarded as background, otherwise the foreground. In the video scenario of the actual ship surveillance, the background contained the water ripple with dynamic shaking, and this single-Gaussian distribution model had no significant effect on dynamic scenario detection. Stauffer $C$ et al., (1998) proposed the Gaussian mixture model (GMM) to replace the single Gaussian distribution to estimate the background [4], and successfully solved the approximate periodic motion of the background, such as the shaking leaves and corrugation, and could also adapt to the change of illumination. Hu et al., (2011) constructed the background image using the median of the first $\mathrm{n}$ frames of images, and proposed the corresponding background updating method to realize the automatic detection of illegal target intrusion in mariculture zone [5]. Borghgraef A et al., (2010) and Wang et al., (2013) pre-introduced target information to be detected [6, 7]. Based on the pixel subtraction of the background to obtain the ship target image, and then to update and assessment. Running Mean Updating Background (RMUB) was used to obtain the foreground image by constructing the background model, reducing the "empty" phenomenon in moving target detection [8], but it was sensitive to illumination change, while initializing the update background phase was slow, background 
learning was lagged behind, causing the foreground target be detected to background. Cai et al., (2015) could reach real-time pedestrian detection based on the calibration of side-ofpavement line and saliency texture detection [9]. However, there was usually no the calibration of side at sea, and it was difficult to reach real-time target detection of moving ship. In the fixed background, the monocular vision is used to generate probably existing area of target. The binocular vision is used to eliminate error detection. However, moving ship target detection environment often contains dynamic shaking water ripples, it is easy to see the background area as probably existing area of target. Wang et al. (2005) [10] proposed a maritime video detection method according to the position of pixel to choose the number of Gaussian distributions. In the actual video surveillance scene of the moving ship target, it is very easy to take the background for the foreground.

The ship monitoring video scenario is complex, the outside weather and background water ripples have significant influence on it. In view of the above analysis, GMM can adapt to the changing of complex background. Therefore, GMM is used to detect the target of moving ship. Finally, the reliability of the algorithm will be verified by experiments.

\section{MOVING SHIP TARGET DETECTION}

The video image of the moving ship are collected by the infrared thermal imager or infrared high definition camera installed on the ship, gate, bridge and so on. It usually includes four parts: sky, ship, sea surface and fixed object. Among them, the sky, sea and fixed objects are marked as the background, ship is marked as the foreground. In this paper, the GMM is used to detect the target of moving ship. The detection includes background modeling, model training and updating, the elimination of shadow and the segmentation of foreground. Specific testing process is shown in Figure 1.

\section{A. Background Modeling}

GMM is a parameterized model, which can approximate the probability density distribution of arbitrary shape, fitting the background distribution of the real scene, and it is widely used in moving target detection [11]. Capturing the continuous video frame image with the change of pixel value $x^{t}$ at the same position in the image of $t$ time, which is considered as a stochastic process. $K$ Gaussian functions are used to fit the gray distribution of the pixel value $x^{t}$. The background model of multiple Gaussian distributions is used to approximate the real background distribution, the probability function is:

$$
\left\{\begin{array}{l}
P\left(X^{t}\right)=\sum_{k=1}^{K} \omega_{k}^{t} \eta\left(X^{t}, \mu_{k}^{t}, \sum_{k}^{t}\right) \\
\eta=1 /\left((2 \pi)^{1 / 2}|\Sigma|^{1 / 2}\right) \exp ^{-\frac{1}{2}\left(X^{t}-\mu_{k}^{t}\right)^{T} \Sigma^{-1}\left(X^{t}-\mu_{k}^{t}\right)}
\end{array}\right.
$$

Where: $P\left(X^{t}\right)$ is the probability function of $X^{t}$; covariance matrices $\sum_{k}^{t} ; \eta$ is The probability density function of the Gaussian distribution; $\mu_{k}^{t}$ is the mean of component $k$ at $t$ time; $\omega_{k}^{t}$ is weight of component $k$ at $t$ time; $K$ is number of mixture components, $K=3$.

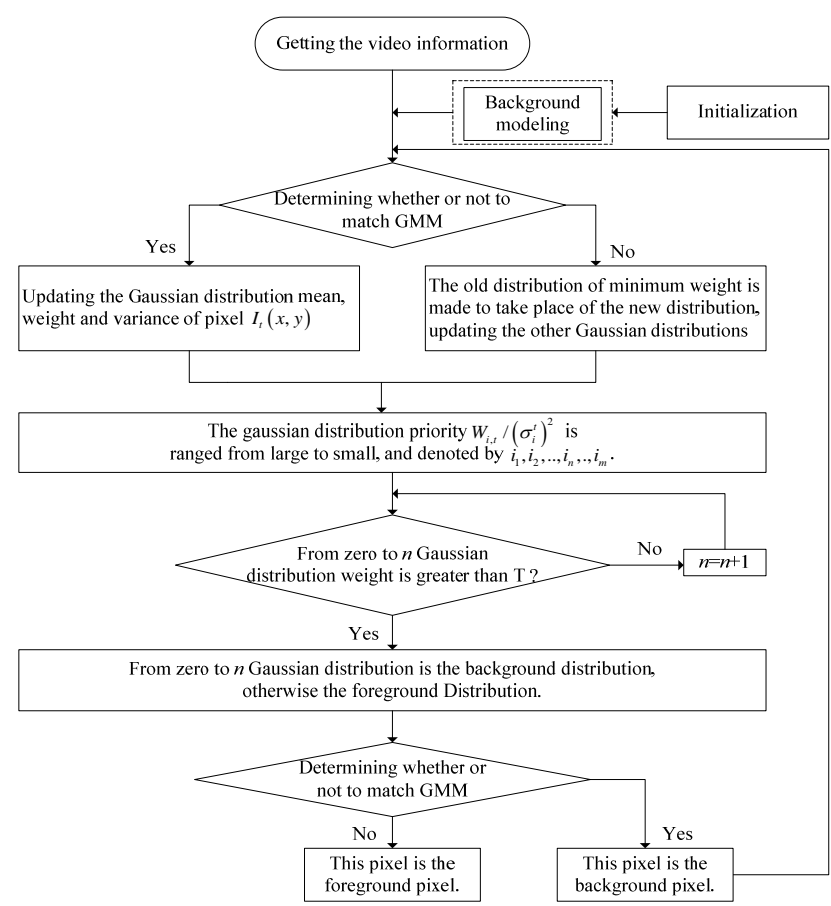

FIGURE I. THE FLOW CHART OF MOVING SHIP DETECTION BASED ON GMM

\section{B. Model Training and Updating}

Model matching is to compare the pixel point $I(x, y)$ observed in the current frame image with the established GMM. If the Eq. 2 is satisfied, it is considered that the pixel point $I(x, y)$ of the current frame matches the established GMM, otherwise, the pixel does not match.

$$
\left|I^{t}(x, y)-\mu_{k}^{t-1}\right| \leq 2.5 \sigma_{k}^{t-1}
$$

Where: $\sigma_{k}^{t-1}$ is the variance of component $k$ at $t-1$ time.

Model matching must update model parameters in real time, the update method sees the Eq. 3 .

$$
\left\{\begin{array}{l}
\mu_{k}^{t+1}=(1-\alpha) \mu_{k}^{t}+\sigma_{k}^{t} x^{t} \\
\sigma_{k}^{t+1}=(1-\rho) \sigma_{k}^{t}+\rho\left(\mu_{k}^{t}-x^{t}\right)^{2} \\
\omega_{k}^{t+1}=(1-r) \omega_{k}^{t}+r M_{k}^{t}
\end{array}\right.
$$

Where: $\alpha$ is update parameter, according to [12], the value is $0.02 ; \rho \approx \alpha / \omega ; r$ is updated parameter for weight; $M_{k}^{t}$ is a value that model matched is 1 , otherwise 0 .

Keeping 3 Gauss components arrange from big to small. 
When the model does not match, the current frame pixel appears new distribution, and the mean $\mu_{k}^{t}$ of the current frame is used; besides, initializing a larger variance and a smaller weight. The new Gaussian distribution model replaces the last Gaussian distribution in the original sequence, and the remaining two Gaussian distributions keep the same mean and variance. Updating the weight according to Eq. 4.

$$
\omega_{k}^{t+1}=(1-r) \omega_{k}^{t}
$$

\section{The Segmentation of Foreground}

In the GMM model parameter learning phase, the Gaussian distribution with a large weight describes a large probability of background pixel value, otherwise a small probability of foreground pixel value. The Gaussian distribution is sorted by the value of $\omega_{k}^{t} / \sigma_{k}^{t}$ from big to small. If $i_{1}, i_{2}, \ldots, i_{n}, \ldots, i_{m}$ means that each Gaussian distribution are arranged according to the value of $\omega_{k}^{t} / \sigma_{k}^{t}$ from big to small at $t$ time and the first $n$ Gaussian distributions are satisfied with Eq. 5, then first $n$ Gaussian distributions are the background distribution and the rest are the foreground distributions.

$$
\sum_{k=1}^{i_{n}} \omega_{k}^{t} \geq T
$$

Where: $T$ general value is $0.7 \sim 0.8$, here is 0.75 .

\section{THE EXPERIMENTAL RESUlT AND ANALYSIS}

\section{A. The Performance Evaluation of Algorithm Detection}

In order to evaluate the detection performance of GMM, the target precision rate (TPR) and false alarm probability (FPR); Zhang et al., (2017) are selected as the evaluation criterion [12].

$$
\left\{\begin{array}{l}
P_{F P R}=N_{F P} /\left(N_{F P}+N_{T N}\right) \\
P_{T P R}=N_{T P} /\left(N_{T P}+N_{F N}\right)
\end{array}\right.
$$

Where: “ $N_{T P}$ ” are the number of times that the target of a moving ship is detected in the target area. $N_{F N}$ ” are the number of times that the target of a moving ship is not detected in the target area. " $N_{F P}$ ” are the number of times that the target of a moving ship is detected in the background area. “ $N_{T N}$ ” are the number of times that the target of a moving ship is not detected in the background area.

\section{B. Experimental Analysis}

In view of different complex sea conditions, in this paper, a large number of experimental tests are carried out to compare GMM with conventional detection method: AFDM and RMUB, to verify the reliability of GMM in the detection of moving ship targets. All experiments are using MATLAB programming language, the computer configuration: Windows10 , Intel(R)1.8GHz, memory 8GB of memory. The resolution of the image: $432 \times 240$.

In the experiments, a total of 536 images of 2 scenarios are selected as experimental test samples. Scenario 1 is a surveillance video with a total of 269 frames, which moving ship target through the ship lock in a relatively fixed background. Scenario 2 is a surveillance video with a total of 267 frames, which indicated sailboat moved from right to left in the condition of small waves.

Figure 2 shows some experimental results in scenario 1. The first column is the original image of frames 60 and 169 in the surveillance video. The second to the fourth column represents that the saliency maps of moving ship extracted using AFDM, RMUB method and GMM. The fifth column indicates the results of using the GMM algorithm for the detection of moving ship targets. In the initial detection stage, the three methods achieve good detection results. The saliency maps are shown in Fig. 2 (b, c, d). With the detection goes deep, the detection results of GMM algorithm are superior to the other two methods, as shown in Figure 2 (g, h, i), owing to the color of a part of the background is similar to the target of a moving ship, the target foreground of a moving ship are mistakenly detected as background in AFDM and RMUB method. The detection rate of the GMM algorithm is $100 \%$, while that of AFDM and RMUB method is $85 \%$ and $98.01 \%$. The result shows that when the background is relatively fixed, the detection rate of the GMM algorithm is higher than that of AFDM and RMUB. 


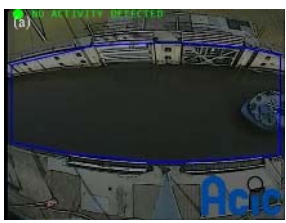

(a)

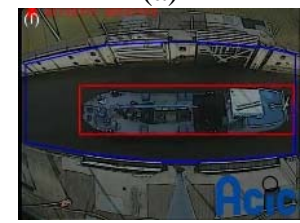

(f)

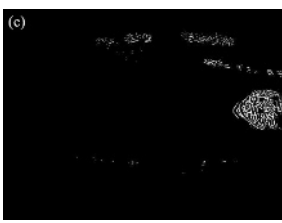

(b)

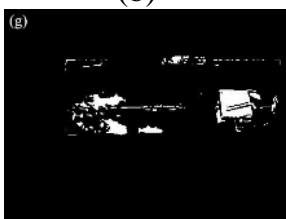

(g)

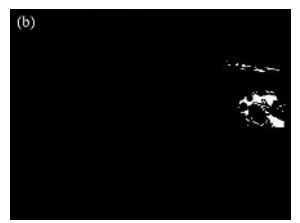

(c)

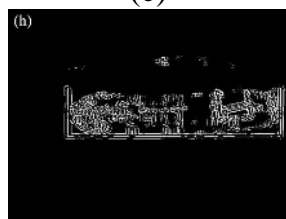

(h)

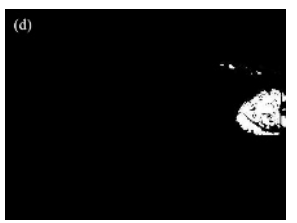

(d)

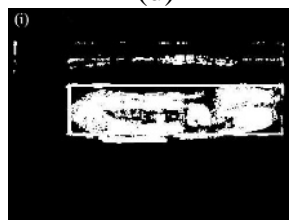

(i)

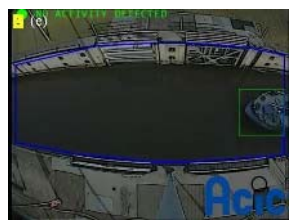

(e)

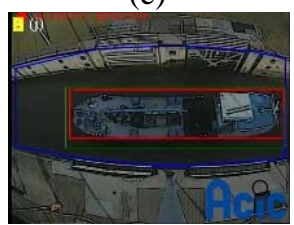

(j)

FIGURE II. DETECTION RESULTS OF THREE METHODS. (A, F)ORIGINAL IMAGES; (B, G) SALIENCY MAPS OF AFDM; (C, H) SALIENCY MAPS OF RMUB; (D, I) SALIENCY MAPS OF GMM; (E, J) DETECTION RESULTS OF GMM.

Figure 3 shows some experimental results in scenario 2. The first column is the original image of frames 80 and 192 in the surveillance video. The second to the fourth column represents that the saliency maps of moving sailing extracted by using AFDM, RMUB and GMM. The fifth column indicates the result that using the GMM algorithm for the detection of moving sailboat target. As shown in Figure 3 (b, c, d), in the background initialization phase, the detection results of GMM and AFDM are better than the RMUB, at the same time RMUB is used to extract the target foreground of the moving sailboat from the surveillance video for twice, which causing the false inspection. With the update of the background model

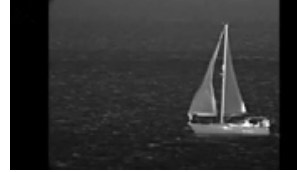

(a)

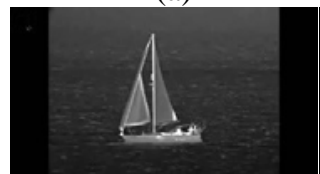

(f)

(f)

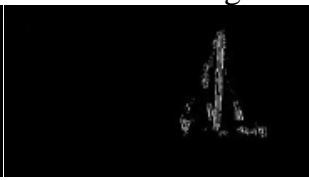

(b)

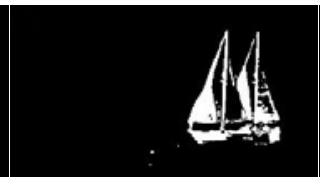

(c) parameters, although the RMUB can get a relatively complete texture region of the moving sailboat, it is difficult to avoid taking the background water ripple as foreground, as shown in Figure 3 (b, g). In the initialization model, GMM has not yet fully learned the complex background, as shown in Figure 3 (d, i), which causing a lot of missing targets. As the detection goes deeper, fewer and fewer targets missing. The results indicate that GMM could deal with some problems such as the similarity between the background color and the moving sailing target color and noise interference caused by waving water ripples in the background.

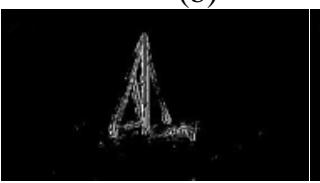

(g)

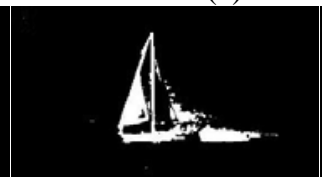

(h)

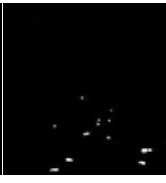

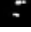

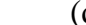

(d)

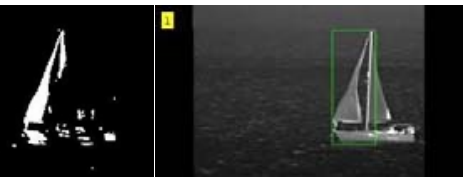

(e)

FIGURE III. DETECTION RESULTS IN THE CONDITION OF SMALL WAVES. (A, F) ORIGINAL IMAGES; (B, G) SALIENCY MAPS OF AFDM; (C, H) SALIENCY MAP OF RMUB; (D, I) SALIENCY MAPS OF GMM; (E, J) DETECTION RESULTS OF GMM

In order to verify the effectiveness of the GMM as a whole, using the AFDM, RMUB and GMM to deal with the monitor video in the three scenarios separately, which is conducted under the same simulation environment. In this paper, we calculate the TPR and FAR of different methods in different scenarios respectively, the results in shown in Table 1.

TABLE I. TPR AND FAR OF DIFFERENT METHODS IN DIFFERENT SCENARIOS

\begin{tabular}{|c|c|c|c|c|c|c|}
\hline \multirow{2}{*}{ Sequence } & \multicolumn{3}{|c|}{$P_{\text {TPR }}$} & \multicolumn{3}{c|}{$P_{F P R}$} \\
\cline { 2 - 7 } & AFDM & RMUB & GMM & AFDM & RMUB & GMM \\
\hline Scenario 1 & $85.00 \%$ & $98.01 \%$ & $100.0 \%$ & $10.21 \%$ & $5.94 \%$ & $6.03 \%$ \\
\hline Scenario 2 & $100.0 \%$ & $99.10 \%$ & $100.0 \%$ & $2.60 \%$ & $1.80 \%$ & $0.00 \%$ \\
\hline
\end{tabular}

As shown in Table 1, GMM is better than AFDM and RMUB. From the statistical results, The TPR of GMM is 100\%, higher than the other two methods. The FPR of GMM is 3.02\%, lower than the other two methods. The reason why there is the FPR is that the ship size is smaller and also there is some significant disturbance that caused by the impact of the sea wave. In complex sea conditions, when background has fixed object marks and shaking water ripples, the performance of GMM algorithm is better than that of the other two methods. 


\section{CONCLUSION}

In this paper, the moving target is detected by ship surveillance video under three scenarios. The detection result shows that the average detection rate of the three methods, AFDM, RMUB and GMM, are similar. GMM is slightly higher than AFDM and RMUB. However, the FPR of the three detection methods is quite different. The FAR of GMM is $3.02 \%$. The FAR of AFDM and RMUB is $6.41 \%$ and $3.87 \%$ respectively. The results show that GMM has better detection results compared with traditional detection method, such as AFDM and RMUB, and has strong anti-interference ability. GMM can achieve good results regardless of whether the background is relatively fixed, or the noise interference caused by light, or the ripple of a large amount of shaking.

The next step is to study the influence of weather condition, blocked moving targets under other conditions, such as poor visibility, etc., to design and develop an easier and more practical background model to reduce the influence of dynamic scenario change on the detection of moving ship.

\section{ACKNOWLEDGEMENTS}

Liaoning natural science foundation of China (20160284); Liaoning natural science foundation of China (201602082); Dalian maritime university teaching reform project funding (2015Y02)

\section{REFERENCES}

[1] LI Cong-sheng, BAI Jun, ZHOU Guang-lu. Vehicle detection of city road at night base on frame difference[J]. Manufacturing automation, 2011, 33(4):212-214

[2] ZHANG Shui-fa, ZHANG Wen-sheng, DING Huang. Background modeling and object detecting based on optical flow velocity field[J]. Journal of image and graphics, 2011, 16(2):236-243.

[3] Friedman N, Russell S. Image segmentation in video sequences: A probabilistic approach[C]//Proceedings of the Thirteenth conference on Uncertainty in artificial intelligence. Morgan Kaufmann Publishers Inc., 1997: 175-181.

[4] Stauffer C, Grimson W E L. Adaptive Background Mixture Models for Real-Time Tracking[J]. Proc Cvpr, 1998, 2:2246.

[5] HU W C, Yang C Y, Huang D Y. Robust real-time ship detection and tracking for visual surveillance of cage aquaculture[J]. Journal of Visual Communication \& Image Representation, 2011, 22(6):543-556.

[6] Borghgraef A, Barnich O, Lapierre F, et al. An Evaluation of Pixel-Based Methods for the Detection of Floating Objects on the Sea Surface[J]. Eurasip Journal on Advances in Signal Processing, 2010, 2010(1):5.

[7] WANG Wei-ye. Moving Object Detection and Behavior Recognition in Intelligent Visual Surveillance[D]. Xian: Xidian University, 2013.

[8] DING Wei. Research And Realization of Vehicle Detection Algorithm in Intelligent Transportation[D]. Nanjing: Nanjing University of Posts Telecommunications, 2012.

[9] CAI Ying-feng, WANG Hai, CHEN Xiao-bo. Vehicle detection and tracking algorithm based on monocular and binocular vision fusion[J]. Journal of Traffic and Transportation Engineering, 2015, 15(6):118-126.

[10] Wang J, Bhat P, Colburn R A, et al. Interactive video cutout[J]. ACM Transactions on Graphics (ToG), 2005, 24(3): 585-594.

[11] REN Ke-Qiang, ZHANG Pang-hua, XIE Bin. Adaptive learning algorithm for moving target detection based on Gaussian mixture model[J]. Computer Engineering and Design, 2014, 35(3):968-974.

[12] Zhang Y, Li Q Z, Zang F N. Ship detection for visual maritime surveillance from non-stationary platforms[J]. Ocean Engineering, 2017, 141: 53-63. 